\title{
ON THE MEASUREMENT OF THE ORIENTATION DISTRIBUTION OF LINEAL AND AREAL ARRAYS*
}

\author{
BY \\ E. M. PHILOFSKY** AND J. E. HILLIARD \\ Northwestern University
}

Introduction. In certain areas of technology it is desirable to have means of specifying the orientation distribution of lineal and areal elements in space. It has been suggested by Hilliard [1] that the orientation distribution of a two-dimensional lineal array be defined by a density function, $L_{A}(\theta)$, where $L_{A}(\theta) d \theta$ is the total line length per unit area to which tangents have angles in the range $\theta \pm d \theta / 2$ with respect to a given reference direction. It will be assumed that the sense of a line element is indeterminate; thus $L_{A}(\theta)$ has a periodicity of $\pi$; i.e., $L_{A}(\theta+n \pi)=L_{A}(\theta)$ for any integral value of $n$. The line length over any finite range of $\theta$ is given by the integral over that range; in particular, the total line length per unit area, $L_{A}$, is

$$
L_{A}=\int_{0}^{\pi} L_{A}(\theta) d \theta .
$$

For the special case when the lineal array is the perimeter of a closed convex figure of area $A$, the density function is also related to the radius of curvature, $\rho$. By definition

$$
L_{A}(\theta) d \theta=d s / A
$$

where $d s$ is the differential of are length. Thus

$$
L_{A}(\theta)=\rho / A \text {. }
$$

Equation (2) can be written in parametric form:

$$
\begin{aligned}
& y(\lambda)=\int_{0}^{\lambda} L_{A}(\theta) \cos \theta d \theta \quad(0 \leq \lambda<\pi) . \\
& x(\lambda)=\int_{0}^{\lambda} L_{A}(\theta) \sin \theta d \theta
\end{aligned}
$$

These equations can be used to construct a lineal array having a given orientation distribution. Since any element of the array can be translated without changing the orientation distribution (a translation corresponds to a change in the lower limit of integration in Eq. (4)) there are an infinite number of arrays satisfying a given distribution function.

Hilliard [1] has also defined density functions for the orientation distribution of three-dimensional lineal and areal arrays in terms of a spherical coordinate system; the orientation of a line segment is denoted by $\phi$ and $\theta$, the longitude and co-latitude of its tangent, and the orientation of an areal element by the angles $\omega$ and $\psi$ of its normal.

${ }^{*}$ Received October 26, 1967.

** Present address: Motorola, Semiconductor Products Division, Phoenix, Arizona. 
The orientation distribution of a lineal array is specified by the function $L_{V}(\phi, \theta)$, where $L_{V}(\phi, \theta) \sin \phi d \phi d \theta$ is the total line length per unit volume in the range $\phi \pm d \phi / 2$ and $\theta \pm d \theta / 2$. The areal distribution can similarly be defined by the function $S_{V}(\psi, \omega)$. The sense of a line or areal element will be assumed indeterminate; hence, all angles specifying orientation will be confined to a hemisphere having the range $0 \leq(\theta, \omega)<2 \pi$ and $0 \leq(\phi, \psi)<\pi / 2$. The line length over any finite interval of $\theta$ or $\phi$, or the boundary area over any finite interval of $\omega$ and $\psi$, will be given by the integral over the range; in particular, the total line length per unit volume is

$$
L_{V}=\int_{0}^{2 \pi} \int_{0}^{\pi / 2} L_{V}(\phi, \theta) \sin \phi d \phi d \theta,
$$

and the total boundary area per unit volume is

$$
S_{V}=\int_{0}^{2 \pi} \int_{0}^{\pi / 2} S_{V}(\psi, \omega) \sin \psi d \psi d \omega
$$

Measurement of the orientation distribution of two-dimensional lineal arrays. In principle, $L_{A}(\theta)$ can be found by dividing the lines in the array into equal segments short compared to the radius of curvature, and then measuring the number of segments as a function of orientation. However, this procedure is infeasible except for a simple array. An alternative procedure is based on the additional number of intersections created when a "test" array of known orientation distribution, $L_{A}^{\prime}(\omega)$, is superimposed on the lineal array defined by $L_{A}(\theta)$; the angles $\theta$ and $\omega$ are referred to separate reference directions in the two arrays.

Hilliard [1] has shown that the expected number of intersections per unit area, $\bar{P}_{A}(\mu)$, created when the two arrays are superimposed randomly with respect to translation but with a specified angle $\mu$ between their reference directions is given by

$$
\bar{P}_{A}(\mu)=\int_{0}^{\pi} \int_{0}^{\pi} L_{A}(\theta) L_{A}^{\prime}(\omega)|\sin (\mu+\theta-\omega)| d \theta d \omega .
$$

A solution for $L_{A}(\theta)$ in terms of the measured function $\bar{P}_{A}(\mu)$ has been obtained [1] to this equation when the test array is a grid of parallel lines. We will now derive a general solution for a test array of any form.

Let us expand each function in Eq. (7) in a Fourier scries. Since the functions have a period of $\pi$, only even terms will appear in the expansion. We can therefore write

$$
L_{A}(\theta)=\sum_{n=-\infty}^{\infty} c_{2 n}^{\theta} \exp (i 2 n \theta)
$$

where the coefficients $c_{2 n}^{\theta}$ are given by

$$
c_{2 n}^{\theta}=(1 / \pi) \int_{0}^{\pi} L_{A}(\theta) \exp (-i 2 n \theta) d \theta .
$$

We can similarly expand $L_{A}^{\prime}(\omega)$ and $\bar{P}_{A}(\mu)$ in Fourier series having coefficients $c_{2 n}^{\omega}$ and $c_{2 n}^{\mu}$. The Fourier expansion for the absolute value of $\sin (\mu+\theta-\omega)$ is

$$
|\sin (\mu+\theta-\omega)|=\sum_{n=-\infty}^{\infty} c_{2 n} \exp [i 2 n(\mu+\theta-\omega)],
$$

where $c_{2 n}=2 / \pi\left(1-4 n^{2}\right)$. On substituting these expansions into Eq. (7) and applying 
the multiplication theorem with respect to $\theta$, we obtain

$$
c_{2 n}^{\mu}=\left[2 \pi /\left(1-4 n^{2}\right)\right] c_{2 n}^{\omega} c_{-2 n}^{\theta} ;
$$

or, in terms of sine $\left(b_{2 n}\right)$ and cosine $\left(a_{2 n}\right)$ Fourier coefficients,

$$
\begin{aligned}
& a_{2 n}^{\mu}=\left[\pi /\left(1-4 n^{2}\right)\right]\left(a_{2 n}^{\omega} a_{2 n}^{\theta}+b_{2 n}^{\omega} b_{2 n}^{\theta}\right) \\
& b_{2 n}^{\mu}=\left[\pi /\left(1-4 n^{2}\right)\right]\left(b_{2 n}^{\omega} a_{2 n}^{\theta}-a_{2 n}^{\omega} b_{2 n}^{\theta}\right)
\end{aligned}
$$

If a two-fold symmetry with perpendicular mirror planes (known as $(2 \mathrm{~mm})$ in crystallography) is present in the lineal array [i.e., $L_{A}(-\theta)=L_{A}(\theta)$ ] the $b_{2 n}^{\theta}$ coefficients will vanish when the reference direction is chosen coincident with one of the mirror axes.

The procedure for determining the orientation distribution, $L_{A}(\theta)$, of a lineal array is thus as follows. An array having a known distribution, $L_{A}^{\prime}(\omega)$ is applied at a series of different orientations $\mu$. A count of the number of intersections between the two arrays yields the function $\bar{P}_{A}(\mu)$ from which can be computed $a_{2 n}^{\mu}$ and $b_{2 n}^{\mu}$. Substituting these coefficients in Eq. (11) together with the known $a_{2 n}^{\omega}$ and $b_{2 n}^{\omega}$ coefficients, one obtains the coefficients of the function $L_{A}(\theta)$.

It is interesting to consider a set of test arrays for which the coefficients of the orientation distribution satisfy the condition

$$
\begin{array}{ll}
a_{2 n}^{\omega}=0 & \text { except for } n=k, \\
b_{2 n}^{\omega}=0 & \text { for all } n,
\end{array}
$$

where $k$ is a given integer or 0 . For $k>0$, an array satisfying Eq. (12) will consist of equal lengths of what may be regarded as positive and negative segments. For $k=0$ all segments of the array are positive. When such arrays are applied to an unknown array at $\mu=0$, it follows from Eq. (11) that the expected net number of intersections per unit area (defined as the number of intersections on positive segments less the number on negative segments) is given by

$$
\bar{P}_{A}= \begin{cases}(\pi / 2) a_{0}^{\omega} a_{0}^{\theta}, & \text { for } \quad k=0, \\ {\left[\pi /\left(1-4 k^{2}\right)\right] a_{2 k}^{\omega} a_{2 k}^{\theta}} & \text { for } \quad k>0 .\end{cases}
$$

This can be rewritten in terms of the number of intersections per unit length of the test array. We first note that the total length (disregarding the signs of the segments) of the test array is, according to Eq. (1),

$$
L_{A}= \begin{cases}\int_{0}^{\pi}(1 / 2) a_{0}^{\omega} d \omega=(\pi / 2) a_{0}^{\omega}, & \text { for } k=0, \\ \int_{0}^{\pi} a_{2 k}^{\omega}|\sin 2 k \omega| d \omega=2 a_{2 k}^{\omega}, & \text { for } k>0 .\end{cases}
$$

Thus, dividing Eq. (13) by (14), we obtain for the expected net number of intersections per unit length of the test array

$$
\bar{P}_{L}= \begin{cases}a_{0}^{\theta} & \text { for } k=0, \\ {\left[\pi / 2\left(1-4 k^{2}\right)\right] a_{2 k}^{\theta}} & \text { for } \quad k>0 .\end{cases}
$$

Thus, by the use of the appropriate test array, it is possible to determine directly any given Fourier component of the orientation distribution of the unknown array. 

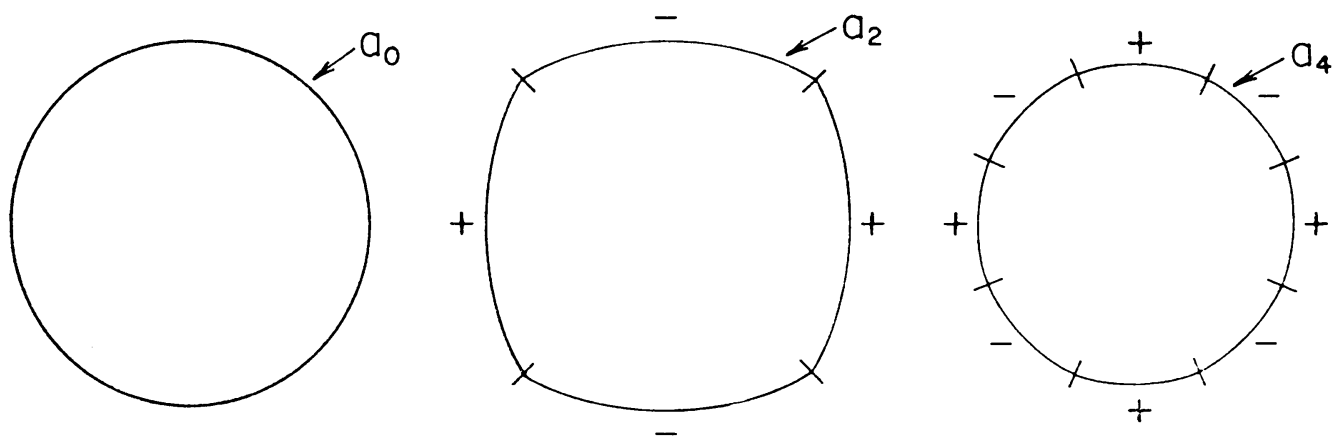

FIg. 1. The perimeters of these figures have orientation distributions satisfying the relationship $L_{A}{ }^{\prime}(\omega)=$ $L_{A} \cos 2 k \omega$ with $k=0,1$ and 2 . When applied to a lineal array, the net number of intersections is directly proportional to the $k$ th Fourier coefficient of that array.

It follows from Eqs. (4) and (12) that one possible test array for $a_{0}^{\omega}$ is a circle. The higher-order arrays in the form of closed convex figures can be constructed from the parametric equations

$$
\begin{aligned}
& x(\lambda)=[\sin (1-2 k) \lambda+\cos (1+2 k) \lambda] / 2\left(1-4 k^{2}\right), \quad \text { for } \quad k>0 . \\
& y(\lambda)=-[\cos (1-2 k) \lambda+\cos (1+2 k) \lambda] / 2\left(1-4 k^{2}\right)
\end{aligned}
$$

Test arrays for $k=0,1$, and 2 are illustrated in Fig. 1 .

The following are some of the properties of these special test arrays:

(1) For any given coefficient, there are an infinite number of possible figures since, as previously noted, any element can be translated without changing the orientation distribution. For example, possible test arrays for $L_{A}^{\prime}(\omega)=L_{A} \cos 2 \omega$ are illustrated in Fig. 2.

(2) There exist a second set of arrays for estimating the sine coefficients, $b_{2 k}^{\theta}$. These are of the same form as the cosine arrays but are rotated by $(\pi / 2 k)$.

(3) Test arrays for $k>0$ in the form of closed convex figures consist of $2 k$ curved arcs of alternating signs. The curvature of the arcs decreases with increasing $k$.
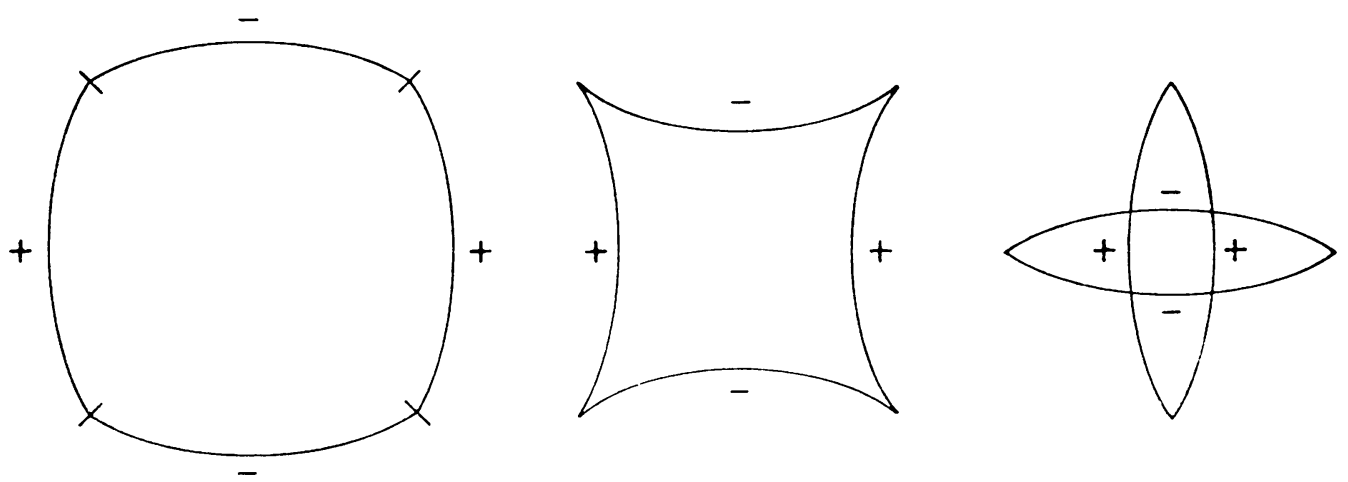

FIG. 2. Three of the possible test figures having perimeters with the orientation distribution $L_{A}{ }^{\prime}(\omega)=$ $L_{A} \cos 2 \omega$. 
(4) The figures are orthogonal in the sense that the expected net number of intersections is zero if two figures with different coefficients are superimposed.

Measurement of the orientation distribution of three dimensional lineal and areal arrays : general case. Let us consider an imaginary experiment in which an areal and a lineal array are "superimposed" on one another. The two arrays will interact to form point intersections. We will first derive an expression for the number of intersections per unit volume and then show how this quantity can be used to determine the orientation distribution of one of the arrays. The first step in the derivation has been given elsewhere [1] but, for the convenience of the reader, it will be summarized here.

Let $L_{V}(\phi, \theta)$ and $S_{V}(\psi, \omega)$ be the orientation distribution of the lineal and areal arrays (as defined earlier) and let the orientations in the two arrays be referred to the same set of axes. We wish to determine the expected number, $\bar{P}_{V}$, of point intersections per unit volume generated when the two arrays are superimposed randomly with respect to translation. Without any loss in generality, we can assume that the line length in the range $\phi \pm(d \phi / 2)$ and $\theta \pm(d \theta / 2)$ occurs as a single segment of length $L_{V}(\phi, \theta) \sin \phi d \phi d \theta$ in a unit volume. We can similarly consider an element of area $S_{V}(\psi, \omega) \sin \psi d \psi d \omega$ in the areal array. For the line segment to intersect the areal element its center must fall within the solid figure generated by the line segment as its center is traversed around the circumference of the areal element. Therefore, if the line segment is randomly located within a unit volume, the probability of it intersecting the areal element is just equal to the volume of the solid figure; namely,

$$
L_{V}(\phi, \theta) S_{V}(\psi, \omega) \sin \phi \sin \psi|\cos \mu| d \phi d \theta d \psi d \omega,
$$

in which $\mu$ is the angle between the line segment and the normal to the areal element and is given by

$$
\cos \mu=\sin \phi \sin \psi \cos (\theta-\omega)+\cos \phi \cos \psi .
$$

The expected total number of intersections per unit volume is found by summing over all orientations in the two arrays. Thus

$$
\bar{P}_{V}=\int_{0}^{\pi / 2} d \phi \int_{0}^{2 \pi} d \theta \int_{0}^{\pi / 2} d \psi \int_{0}^{2 \pi} L_{V}(\phi, \theta) S_{V}(\psi, \omega) \sin \phi \sin \psi|\cos \mu| d \omega .
$$

A solution to this equation for $S_{V}(\psi, \omega)$ or $L_{V}(\phi, \theta)$ in terms of $\bar{P}_{V}$ has been given [1] for special cases. We will now consider the general solution.

The first step is to expand $|\cos \mu|$ in a series of Legendre polynomials. Thus

$$
|\cos \mu|=\sum_{n=0}^{\infty} \alpha_{2 n} P_{2 n}(\cos \mu)
$$

where $\alpha_{2 n}$ are coefficients given by

$$
\alpha_{2 n}=(4 n+1) \int_{0}^{1}(\cos \mu) P_{2 n}(\cos \mu) d(\cos \mu),
$$

which yields $\alpha_{0}=1 / 2, \alpha_{2}=5 / 8$ and

$$
\alpha_{2 n}=(-1)^{n+1}(4 n+1) \frac{1 \cdot 3 \cdot 5 \cdots(2 n-3)}{2 \cdot 4 \cdot 6 \cdots(2 n+2)} \quad \text { for } n=2,3, \cdots .
$$

We next expand $L_{V}(\phi, \theta)$ in a series of spherical harmonics 


$$
L_{V}(\phi, \theta)=\sum_{n=0}^{\infty} Y_{2 n}^{L}(\phi, \theta)
$$

in which

$$
Y_{2 n}^{L}=\sum_{h=-2 n}^{2 n} \gamma_{2 n, h}^{L} P_{2 n}^{\mid h !}(\cos \phi) \exp (i h \theta)
$$

where $P_{2 n}^{h}$ are associated Legendre polynomials and $\gamma_{2 n, h}^{L}$ are coefficients defined by

$$
\gamma_{2 n, h}^{L}=\frac{(4 n+1)}{2 \pi} \frac{(2 n-h) !}{(2 n+h) !} \int_{0}^{2 \pi} \exp (i h \theta) d \theta \int_{0}^{1} L_{V}(\phi, \theta) P_{2 n}^{h}(\cos \phi) d(\cos \phi) .
$$

The function $S_{V}(\psi, \omega)$ can similarly be expanded in a set of harmonics $Y_{2 n}^{S}(\psi, \omega)$. Substituting these series in Eq. (18), we obtain

$$
\begin{array}{r}
\vec{P}_{V}=\int_{0}^{\pi / 2} d \phi \int_{0}^{2 \pi} d \theta \int_{0}^{\pi / 2} d \psi \int_{0}^{2 \pi}\left\{\sum_{n=0}^{\infty}\left[Y_{2 n}^{L}(\phi, \theta) \sin \phi\right] \sum_{n=0}^{\infty}\left[Y_{2 n}^{S}(\psi, \omega) \sin \psi\right]\right. \\
\left.\cdot \sum_{n=0}^{\infty}\left[\alpha_{2 n} P_{2 n}(\cos \mu)\right]\right\} d \omega .
\end{array}
$$

The coefficient $P_{2 n}(\cos \mu)$ is a Laplacian. Thus

$$
\int_{0}^{2 \pi} d \theta \int_{0}^{\pi / 2} Y_{2 n^{\prime}}^{L}(\phi, \theta)(\sin \phi) P_{2 n}(\cos \mu) d \phi=\left\{\begin{array}{l}
0 \quad \text { for } n^{\prime} \neq n, \\
{[2 \pi /(4 n+1)] Y_{2 n}^{L}(\psi, \omega)}
\end{array} \text { for } n^{\prime}=n .\right.
$$

The remaining cross products vanish on integration of Eq. (25) because of the orthogonality of the spherical harmonics. Hence,

$$
\bar{P}_{V}=\sum_{n=0}^{\infty} \sum_{n=-2 n}^{2 n}\left[\frac{2 \pi}{(4 n+1)}\right]^{2}\left[\frac{(2 n+h) !}{(2 n-h) !}\right] \alpha_{2 n} \gamma_{2 n, h}^{L} \gamma_{2 n, h}^{S} .
$$

This equation will yield the orientation distribution of an areal array using a lineal array of known orientation distribution. By superimposing this array on the areal array at a sufficient number of different orientations and counting the number of intersections produced, it is theoretically possible to determine by use of Eq. (26) the coefficients of the areal array up to any required degree. Conversely, it is possible to determine the orientation distribution of a lineal array by use of an areal array of known distribution as the test element. It is apparent, however, that a considerable amount of experimental and computational work will be necessary if a high degree of resolution is required in the estimation of the unknown orientation distribution. Fortunately, in practice, certain symmetries are often present which greatly simplify the analysis. We will now consider two such cases.

Measurement of orientation distribution of areal array with (mmm) symmetry. Processes such as forging and rolling on a sample containing internal boundaries can be expected to produce an orientation distribution with a two-fold axis of symmetry and three perpendicular mirror planes. In crystallography this symmetry is described by the notation $(\mathrm{mmm})$. If the $\psi$ axis is set parallel to the intersection of two of the mirror planes, then

$$
S_{V}(\psi, \omega)=S_{V}[\psi,(\pi-\omega)]=S_{V}[\psi,(\pi+\omega)]=S_{V}[\psi,(2 \pi-\omega)] .
$$

Under this condition the coefficients $\gamma_{2 n, h}^{S}$ vanish for odd values of $h$ and $\gamma_{2 n, h}^{S}=\gamma_{2 n,-h}^{S}$ 
for all values of $h$. Thus the number of coefficients required to describe the orientation distribution is reduced by a factor of four.

Measurement of orientation distribution of an areal array having an axis of symmetry. Deformation by wire drawing, extrusion, etc. results in an areal array having an axis of symmetry. Such symmetry is also often found in nature. If the $\psi$ axis is chosen coincident with the axis of symmetry, the orientation distribution will be independent of $\omega$. Thus $S_{V}(\psi, \omega)=S_{V}(\psi) / 2 \pi$. Under this condition, the associated Legendre polynomial coefficients vanish from the expansion of $S_{V}(\psi, \omega)$, and Eq. (26) reduces to

$$
\bar{P}_{V}=\sum_{n=0}^{\infty}[2 \pi /(4 n+1)]^{2} \alpha_{2 n} \alpha_{2 n}^{L} \alpha_{2 n}^{S}
$$

in which

$$
\alpha_{2 n}^{S}=[(4 n+1) / 2 \pi] \int_{0}^{2 \pi} d \omega \int_{0}^{1} S_{V}(\psi, \omega) P_{2 n}(\cos \psi) d(\cos \psi),
$$

and $\alpha_{2 n}^{L}$ is similarly defined with respect to the function $L_{V}(\phi, \theta)$. The unknown coefficients $\alpha_{2 n}^{S}$ can be determined from an application of a two-dimensional lineal array to a single section taken through the areal array parallel to the axis of symmetry.

As in the two-dimensional case, it is possible to derive test figures for which the intercept density will be directly proportional to a given Legendre polynomial coefficient of the areal array. Such a test figure satisfies the condition

$$
\alpha_{2 n}^{L}=\left\{\begin{array}{l}
L_{V} / 2 \pi \quad \text { if } n=k, \quad(k=0,1,2 \cdots), \\
0 \quad \text { if } n \neq k,
\end{array}\right.
$$

in which $L_{V}$ is the total line length of the test figure per unit volume. If this test figure is applied to a section parallel to the axis of symmetry, then Eq. (27) reduces to

$$
\bar{P}_{V}=2 \pi \alpha_{2 k} \alpha_{2 k}^{S} L_{V} /[4 k+1]^{2},
$$

which can be rewritten

$$
\alpha_{2 k}^{\mathcal{S}}=[4 k+1]^{2} \bar{P}_{V} / 2 \pi \alpha_{2 k},
$$

in which $\bar{P}_{V}$ is the expected number of intersections per unit length of the test figure. It follows from Eqs. (22) and (24) that the lineal test figure for determining the $2 k$ th coefficient will have an orientation distribution given by

$$
L_{V}(\phi, \theta)=L_{V} P_{2 k}(\cos \phi) \delta(\theta) \sin \phi .
$$

The test figures can be constructed from the following pair of parametric equations:

$$
\begin{aligned}
& x(\lambda)=\int_{0}^{\lambda} P_{2 k}(\cos \phi) \cos \phi \sin \phi d \phi \\
& y(\lambda)=\int_{0}^{\lambda} P_{2 k}(\cos \phi) \sin ^{2} \phi d \phi
\end{aligned}
$$

Examples of the figures for determining the $\alpha_{0}^{S}, \alpha_{2}^{S}$, and $\alpha_{4}^{S}$ coefficients are shown in Fig. 3. It should be noted that these figures have properties identical to those of the Fourier figures with respect to signs on the individual arcs, translation of the arcs, and orthogonality. 

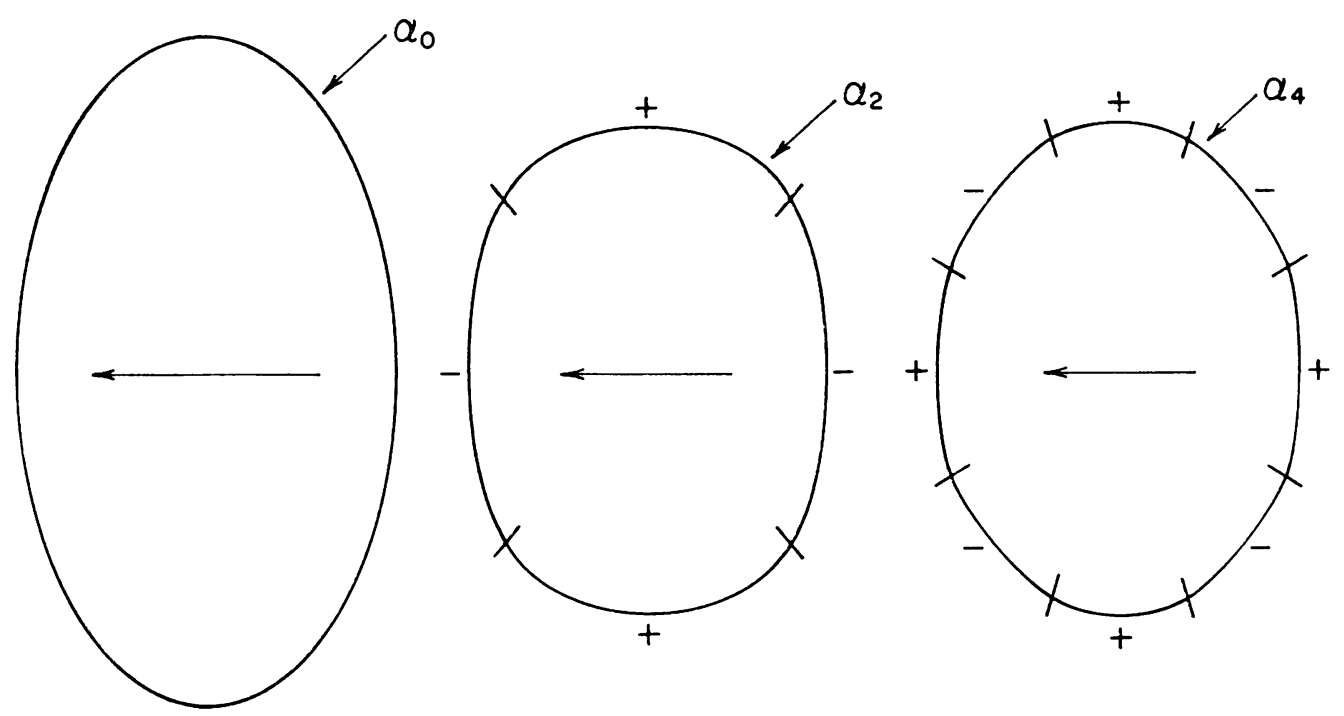

Fig. 3. The perimeters of these figures have orintation distributions satisfying Eq. (30) with $k=0$, 1 and 2. These can be used to determine the corresponding Legendre polynomial coefficients of an areal array having an axis of symmetry. The figures are applied to any section parallel to axis of symmetry.

Applications of the analyses. There are several obvious applications of the foregoing results to the analysis of the microstructure of materials. The distribution functions in three dimensions provide a specific description of the anisotropy of lineal features such as dislocations and of areal arrays such as grain boundaries and magnetic domain walls. The orientation distribution of lineal arrays in two dimensions can be applied to the analysis of dislocation networks as seen by electron transmission microscopy.

A further application is the determination of plastic strains produced by mechanical deformation. By comparing the orientation distribution of grain boundaries before and after deformation, it is possible to estimate strains through the interior of the material [2].

Procedures for optimizing the analyses and an estimate of the sampling errors in the Fourier or spherical harmonic coefficients will be given elsewhere [3].

Acknowlegment. This work was supported by the Advanced Research Projects Agency of the Department of Defense. We are indebted to Professor I. V. Stakgold for a critical review of the manuscript.

\section{REFERENCES}

1] J. E. Hilliard, Trans. Met. Soc., AIME, 224 (1962), 1201.

[2] J. E. Flinn and E. M. Philofsky, Trans. ASM (in press)

[3] E. M. Philofsky and J. E. Hilliard (to be published) 